IRA-International Journal of Technology \& Engineering ISSN 2455-4480

Proceedings of the

International Conference on Science \& Engineering for Sustainable Development (2017)

Pg. no. 1-10

Published by: Institute of Research Advances https://research-advances.org/index.php/IRAJTE

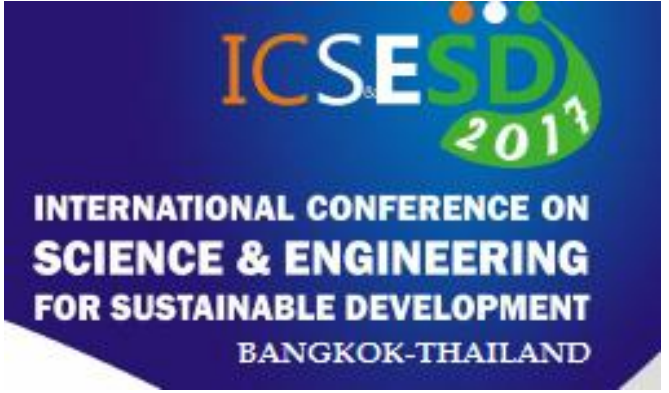

\title{
Analysis of SCERP: A Cluster Based Routing Protocol for Energy Balancing in Wireless Sensor Network
}

\author{
M.A.Pund ${ }^{1}$, Shital V. Bahale ${ }^{*^{2}}$, Jaya Ingole ${ }^{3}$ \\ ${ }^{1}$ Associate Professor PRMIT\&R, Badnera, India. (mapund@mitra.ac.in) \\ ${ }^{2}$ PG Studnet, CSE, PRMIT\&R, Amravati, India. \\ ${ }^{3}$ Associate Professor, PRMITR, India. (jayaingole@yahoo.com)
}

Type of Review: Originality Check \& Peer Review under the responsibility of the Scientific Committee of the Conference and The Institution of Engineers (India). DOI: http://dx.doi.org/10.21013/jte.ICSESD201701

\section{How to cite this paper:}

Pund, M., Bahale, S., Ingole, J. (2017). Analysis of SCERP: A Cluster Based Routing Protocol for Energy Balancing in Wireless Sensor Network. Proceedings of the International Conference on Science \& Engineering for Sustainable Development (2017), 1-10. doi: http://dx.doi.org/10.21013/ite.ICSESD201701

(C) International Conference on Science \& Engineering for Sustainable Development \& The Institution of Engineers (India).

\section{(cc) EY-NO}

This work is licensed under a Creative Commons Attribution-Non Commercial 4.0 International License subject to proper citation to the publication source of the work.

Disclaimer: The conference papers as published by the Institute of Research Advances (IRA) are the views and opinions of their respective authors and are not the views or opinions of the IRA. The IRA disclaims of any harm or loss caused due to the published content to any party. 


\section{ABSTRACT}

Prolonging lifetime of wireless sensor network is a most significant problem due to energy constraint nature of sensor nodes. It is difficult to recharge nodes during network lifetime, to increase application area of WSN there is a need to design energy efficient clustering protocol for WSN. In this article there is a discussion about problems in Leach protocol and propose an improvement on the Leach routing protocol to reduce energy consumption and to extend network lifetime. Proposed self organized cluster based energy balanced routing protocol (SCERP) selects a cluster head node by considering probability based on ratio of residual energy of the node and the average energy level of nodes in network, and the geometric distance between the candidate node to the BS as key parameters. The outcome of simulation shows that proposed protocol is better than Leach in terms of balancing energy consumption of nodes and extending WSN lifetime.

Keywords: Hierarchical Routing Algorithms, LEACH, Cluster-based Routing.

\section{Introduction:}

In general sensors in wireless sensor networks, is a device with capabilities of sensing, processing and transmitting/ receiving. Small batteries are used to fulfill the power source requirement of each node for doing all these operations. However, these batteries must be smart enough to limit use of power so that nodes lifetime can be extended despite of being smaller in size, is one of the wireless sensor networks key challenge[15]. It is a matter of concern for scientists and researchers to focus on development of such protocols that can maximize network lifetime out of limited power source. Another problem is transmission of bulk of sensed information from each node so there is a need of efficient routing protocol that has low routing overhead and well organized data aggregation mechanisms in order to design an efficient wireless sensor network [16].

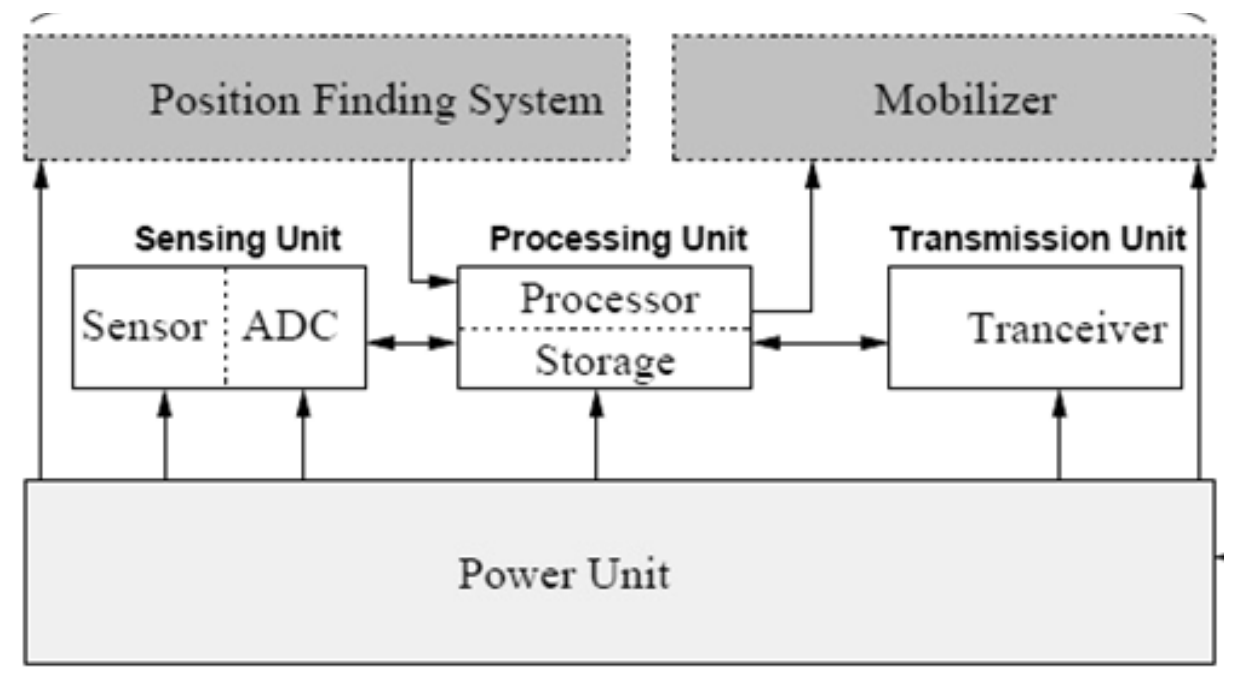

Fig. 1 Components of Sensor Node

Many conventional cluster based routing protocols developed for WSN $[3,4]$ such as Leach[1,6], threshold-sensitive energy efficient sensor network (TEEN) [10], and power efficient gathering in sensor information systems (PEGASIS) [8] and Leach-C. These algorithms need to operate with global knowledge of the whole network, and critical node failure will be a serious issue [2]. A periodically chosen cluster-heads, in the sensor network considered here aggregates and transmit the data received from cluster members to the sink. 
This article, presents an improved clustering mechanism of the LEACH routing protocol for energy efficiency. The proposed routing protocol SCERP provides resolutions two problems in existing LEACH protocol. First, the remaining energy of a candidate node is considered for selecting a cluster head $(\mathrm{CH})$ in order to avoid critical node failure. Second, the shortest geographical distance from the candidate node to the sink is also one of the factors in cluster head selection process. This improvement however checks the remaining energy of existing cluster heads. Cluster heads will remain as cluster head for next round if its remaining energy is greater than the threshold value, it will remain cluster head for the next round as well. This is how, energy wasted in routing packets for new cluster head and cluster formation can be saved. Low energy cluster head having less energy than required threshold, will be replaced with improved cluster head selection formula. MATLAB simulation results shows that under the proposed SCERP protocol network lifetime and energy retention are better than that of LEACH protocol.

The remaining sections of this paper are organized as follows. In Section 2, a brief review of related work is given. Section 3 presents the SCERP algorithm in detail. Section 4 shows the performance of SCERP using MATLAB simulation and compares it with LEACH. Finally, Section 5 gives concluding remarks.

\section{Related Work:-}

LEACH [5] is the clustering algorithms designed for homogeneous network.

The study of Wendi R. Heinzelman proposed LEACH (Low-Energy Adaptive Clustering Hierarchy) protocol points out that in LEACH protocol extra energy is required for periodic election of new cluster heads in each round of operation and for transmitting data to distant sink. A node randomly chooses a number between 0 to 1 at the each stage of cluster forming, then comparison of this number with the threshold values $\boldsymbol{t}(\boldsymbol{n})$ is done, if it found that the number is less than $\boldsymbol{t}(\boldsymbol{n})$, then that node become cluster head for this round, else it become a normal node. Threshold $t(n)$ value is determined by the following formula:

$$
\mathbf{t}(n)= \begin{cases}\frac{p}{1-p^{*}\left(r \bmod \frac{1}{p}\right)} & \text { if } n \in G \\ 0 & \text { if } n \notin G\end{cases}
$$

By analyzing the cluster formation process of LEACH[9] protocol, it is found that it just considers the communication cost between the cluster head and normal node; it fails to consider the remaining energy of cluster head and its location information which results in rapid depletion of network lifetime. On the other hand, random cluster head selection mechanism of LEACH protocol lead to uneven distribution of cluster head node which is main cause behind increase in energy consumption because of increase in transmission distance, thereby affecting the life cycle of the network [7].

The method proposed by Handy MJ, Hasse M, Timmermann D. given in[12] is based on residual energy for cluster head selection. The threshold is calculated as shown in the following equation:

$$
T(n)=\frac{p}{1-p *\left(r \bmod \frac{1}{p}\right)} * \frac{E_{c u r}}{E_{0}}
$$

Ecur is the current energy of node, $E_{0}$ is the initial energy of a node. This method increases the probability of the high-energy nodes to become cluster-head, but it having a significant problem. When 
the nodes remaining energy is very low, the value of threshold $T(n)$ becomes very small. Hence the node random number being smaller than the threshold value probability becomes small. These results in few cluster-head nodes in the network, the energy consumption of selected cluster-heads increases and thus untimely death of nodes affects the network life.

S. Lindsey and C. Raghavendra in [8] introduced PEGASIS (Power Efficient Gathering in Sensor Information Systems) protocol in 2002. Instead of cluster formation, in PEGASIS the nodes will be organized to form a chain. The node is responsible for routing the aggregated information to the sink. Every node passes the aggregated data with its information to the next ring. It employ a multi hop transmission and selects only one node to transmit to the sink. But it requires global knowledge of entire network which makes implementation difficult to implement. Moreover, the excessive amount of energy is consumed in long-distance communication from a node to the sink.

The protocol proposed by A. Manjeshwar and D. P. Agarwal in 2001 [13] is Threshold sensitive Energy Efficient sensor Network Protocol (TEEN). In TEEN nearer nodes form clusters, and a cluster heads transmit the collected information to the higher layer. After cluster formation the cluster heads broadcast 2 threshold values, 1 st is hard threshold and $2^{\text {nd }}$ is soft threshold. Hard threshold is the value that permits transmit event of nodes, if the event happens within the range of interest. Thus there is a reduction in the transmission delay. A node can send a new packet only if amendment of minimum soft threshold happens. This prevents redundant data transmission. Since the protocol is sensitive to the rapid changes in the predefined threshold values; therefore, it is most appropriate for time-critical applications.

\section{Proposed Scheme:-}

Basically, at every round LEACH protocol changes the cluster head and that node will not be considered for next $1 / \mathrm{p}$ rounds. $\mathrm{LEACH}$ protocol requires a lot of energy in each round, for cluster formation and for cluster head replacement. So in the proposed method an energy efficient cluster head replacement scheme is introduced. In this method if the energy of existing cluster head is below certain threshold value it will be replaced with a new one. This saves the energy wastage in packet routing for new cluster head and cluster formation. Besides limiting energy utilization in cluster formation, the improved algorithm considers the distance between normal node and the base station. The smaller distance node to the sink is considered in cluster head election process. The improved threshold in this paper is:

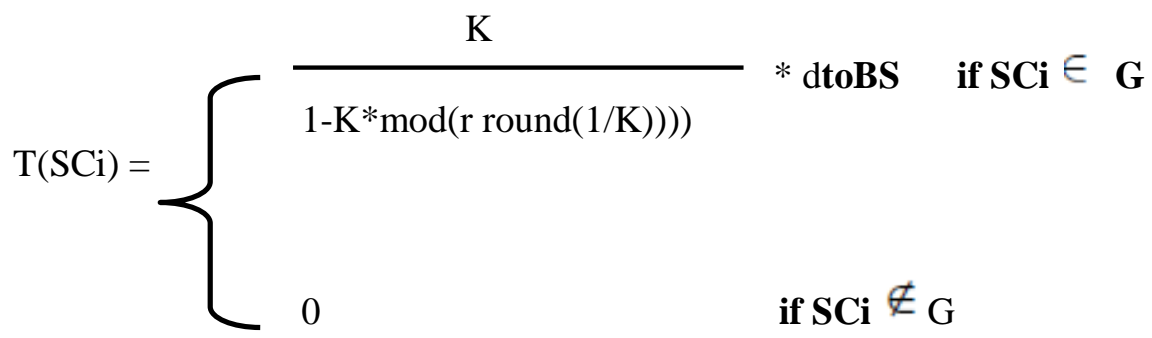

$\mathrm{N}$

Where $\mathrm{K}$ is $\quad \mathrm{K}=\sum_{\mathrm{i}=1} \mathrm{n} * \mathrm{P} *($ Ecur $/ \mathrm{Ea})$ if SCi $\notin \mathrm{G}$

Ecur is the current residual energy of the node, and Ea is the average network energy. The distance between nodes and the base station is presented by dtoBS. 
The proposed algorithm improved the network lifetime, reduces the energy consumption of the network. The proposed protocol design is implemented using MATLAB and we get comparative graph analysis between LEACH and proposed protocol based on energy consumed in network, when first and last node dies and packets transmitted to BS.

\section{Simulation and Results:-}

In order to implement the proposed algorithm simulation parameters are listed in Table 1. The goal is to compare the performance of these algorithms and the level of energy it attends after a certain number of rounds.

Table 1: Simulation Parameters

\begin{tabular}{|c|c|}
\hline Parameter & Value \\
\hline $\operatorname{Area}(x, y)$ & 100,100 \\
\hline Base Station(x,y) & 50,50 \\
\hline Nodes(n) & 100 \\
\hline Probability $(\mathrm{P})$ & 0.1 \\
\hline Initial Energy(Eo) & 0.5 \\
\hline Maximum Lifetime & 5000 \\
\hline Transmitter Energy & $50 \mathrm{~nJ} / \mathrm{bit}$ \\
\hline Receiver Energy & $50 \mathrm{~nJ} / \mathrm{bit}$ \\
\hline Free space amplifier & $10 \mathrm{pJ} / \mathrm{bit} / \mathrm{m}^{2}$ \\
\hline Multipath amplifier & $0.0013 \mathrm{pJ} / \mathrm{bit} / \mathrm{m}^{4}$ \\
\hline Message size & 4000 bits \\
\hline Data aggregation & 5nJ/bit/signal \\
\hline
\end{tabular}

Simulation of Leach and proposed SCERP protocol is done based on the basis of parameters given in the table.

The comparative result analysis of LEACH and SCERP protocol is done for following aspects: network lifetime in terms of first and $90 \%$ dead nodes and energy retention.

Considering network life time of LEACH and SCERP the proposed scheme the LEACH has lowest performance with respect to network life time. Due to its energy efficient cluster head replacement method the proposed scheme has greater stable period [14]. The stable period of lifecycle percentage in Leach Protocol is $8 \%$, the one in the improved protocol is $23 \%$. The stable period of lifecycle percentage in improved algorithm increases by $15 \%$.

Simulated results depicted in figure 2 represent network life time by showing First dead node and when 90\% nodes dies. In Leach protocol the first node died in 439 rounds and $90 \%$ nodes died at 902 round whereas in proposed protocol the first node died in 1171 rounds and $90 \%$ nodes died at 2216 rounds respectively. 
Figure 3 shows the comparison of energy consumption between the Leach and proposed protocol during the simulation time. $\mathrm{X}$ axis represents energy in joules and $\mathrm{Y}$ axis is representing the number of rounds. The energy consumption of the improved algorithm is much lower than that of Leach Protocol for the same round of simulation. There is also a network lifetime comparison between LEACH and proposed system, Table 2 represents round when first and 90\% nodes died in Leach and SCERP protocol at different initial energies, for 100 nodes and 5000 rounds with base station position 50-50.

Table 2. Network Lifetime comparison between Leach and SCERP

\begin{tabular}{|l|l|l|l|l|}
\hline $\begin{array}{l}\text { Initial } \\
\text { Energy }\end{array}$ & $\begin{array}{l}\text { Leach } \\
\text { First Node Dead }\end{array}$ & $\begin{array}{l}\text { SCERP } \\
\text { First Node Dead }\end{array}$ & $\begin{array}{l}\text { LEACH90\% } \\
\text { Nodes Dead }\end{array}$ & $\begin{array}{l}\text { SCERP90\% } \\
\text { Nodes Dead }\end{array}$ \\
\hline 0.1 & 78 & 229 & 198 & 426 \\
\hline 0.2 & 156 & 451 & 370 & 859 \\
\hline 0.3 & 252 & 671 & 533 & 1304 \\
\hline 0.4 & 376 & 958 & 713 & 1799 \\
\hline 0.5 & 439 & 1171 & 902 & 2216 \\
\hline 0.6 & 484 & 1431 & 1051 & 2732 \\
\hline 0.7 & 615 & 1651 & 1281 & 3080 \\
\hline 0.8 & 652 & 1821 & 1403 & 3634 \\
\hline 0.9 & 818 & 2225 & 1590 & 4248 \\
\hline 1.0 & 920 & 2531 & 1719 & 4649 \\
\hline
\end{tabular}

Figure 4 shows network lifetime comparison for varying initial energies of Leach and proposed SCERP protocol. In graph 100 nodes are distributed in the square area $100 * 100 \mathrm{~m}$ with changing initial energies from 0.1 to 1.0. The simulation results show that proposed SCERP protocol performs better than Leach.

Table 3 gives comparative analysis of energy retention in Leach and SCERP protocol at different initial energies verses rounds and at 0.05 cutoff energy.

Table 3. Energy Retention comparison between Leach and SCERP for varying initial energies

\begin{tabular}{|l|l|l|}
\hline $\begin{array}{l}\text { Initial } \\
\text { Energy }\end{array}$ & $\begin{array}{l}\text { Leach Energy } \\
\text { Reaches at cut off } \\
\text { energy 0.05 }\end{array}$ & $\begin{array}{l}\text { SCERP Energy } \\
\text { Reaches at cutoff } \\
\text { energy 0.05 }\end{array}$ \\
\hline 0.1 & 72 & 183 \\
\hline 0.2 & 226 & 548 \\
\hline 0.3 & 385 & 900 \\
\hline 0.4 & 536 & 1309 \\
\hline 0.5 & 692 & 1724 \\
\hline 0.6 & 847 & 2115 \\
\hline 0.7 & 1014 & 2546 \\
\hline 0.8 & 1196 & 3008 \\
\hline 0.9 & 1341 & 3545 \\
\hline 1.0 & 1468 & 3900 \\
\hline
\end{tabular}

Graphical representation shows in figure 5 that proposed SCREP protocol performs much better than Leach protocol and reduces the energy consumption by energy balancing between nodes. 


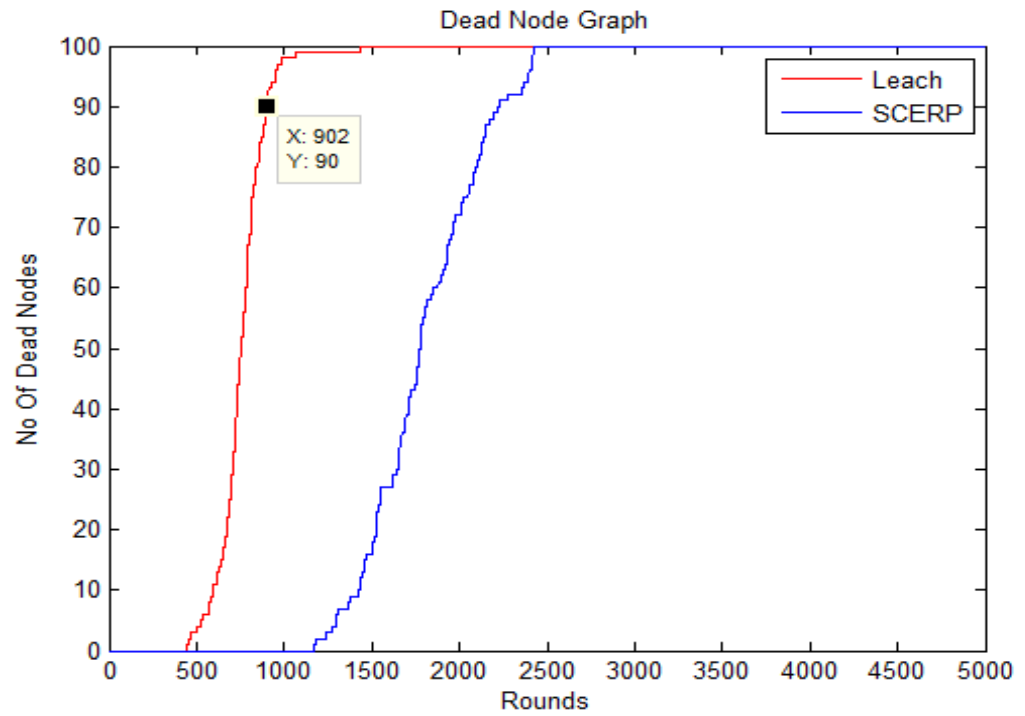

Figure 2 The number of dead nodes per round in Leach and SCERP.(Network Lifetime)

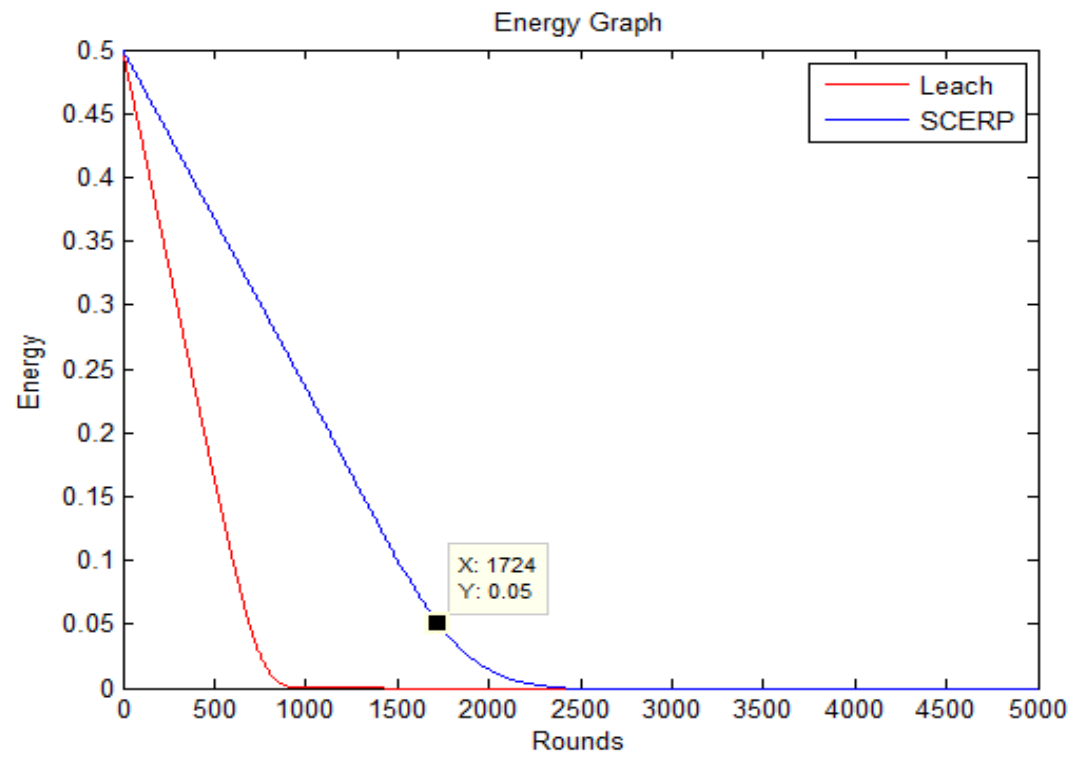

Figure 3 Energy Retention after each round in Leach and SCERP 


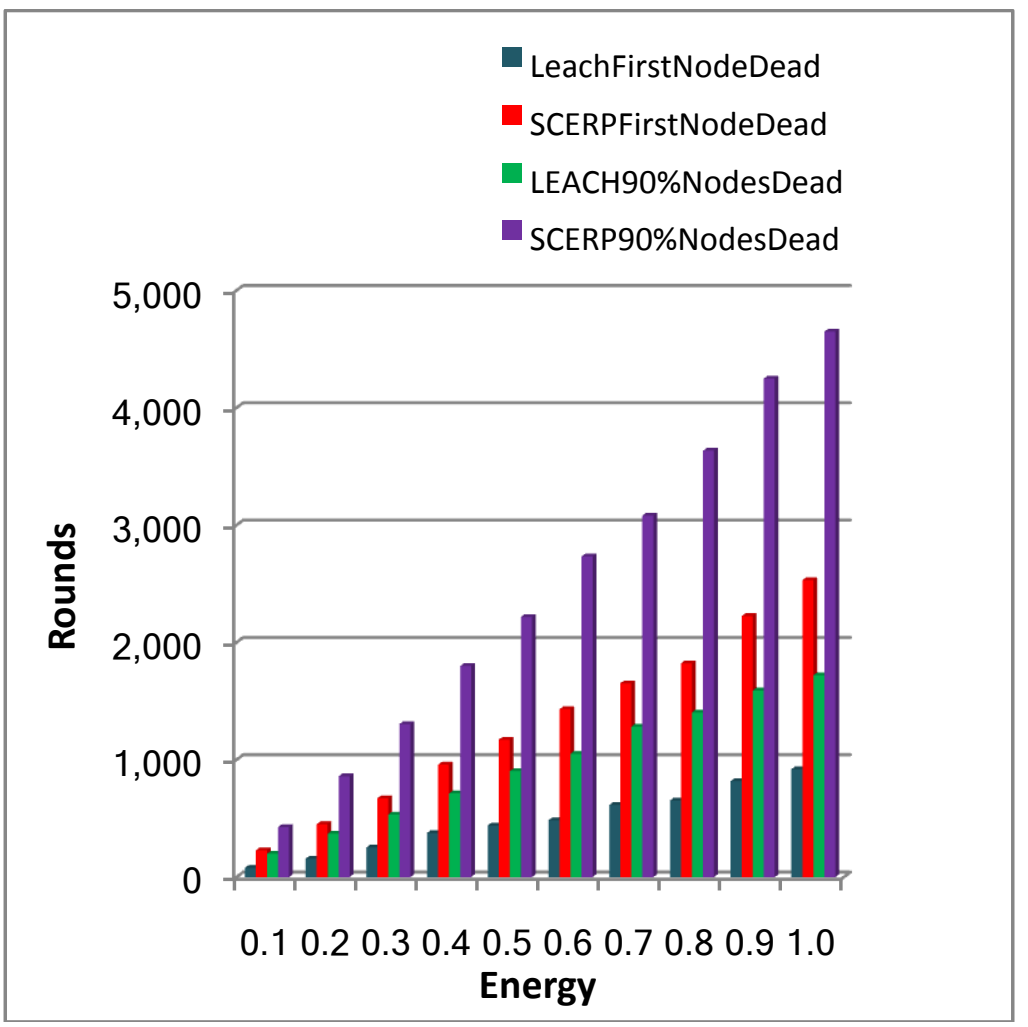

Figure 4 Network Lifetime comparison of Leach \& Proposed System for for varying Initial Energy,100 nodes with Base station at 50,50

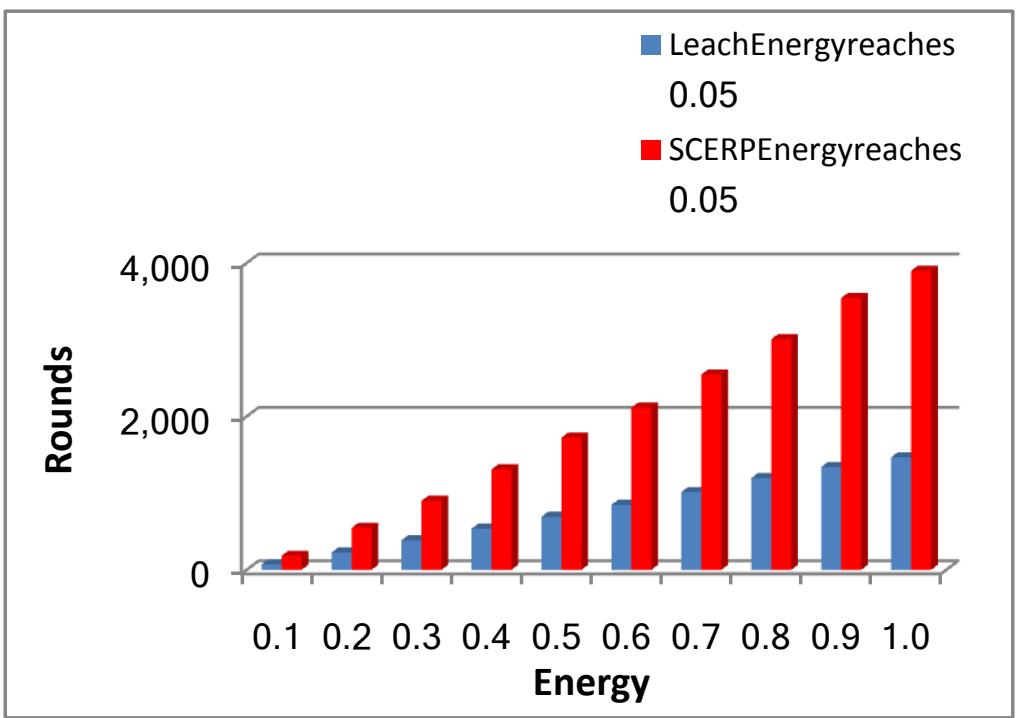

Figure 5 Energy Retention comparison of Leach \& Proposed System for varying Initial Energy,100 nodes with Base station at 50,50 and cutoff energy at 0.05 


\section{Conclusion:}

As the random cluster head election process in LEACH causes quick depletion of nodes energy also it does not consider distance of nodes from base station which imposes heavy energy burden and results in reduction of network lifetime. This paper presents SCERP algorithm to address these issues, which aims at extending network lifetime by balancing energy consumption of sensor nodes. This protocol considers distance and current energy of nodes as factors for selecting cluster heads. The simulation results obtained from Matlab platform shows that the stable period of lifecycle percentage in Leach Protocol is $8 \%$, the one in the improved protocol is $23 \%$, whereas percentage of reliable network for operation in Leach protocol is $18 \%$ and in proposed scheme it is $44.32 \%$. Therefore the proposed protocol outperform LEACH protocol in terms of energy retention and the lifetime of network by using efficient cluster head replacement mechanism.

\section{Reference:}

[1] Ian F. Akyildiz, Weilian Su, Yogesh Sankarasubramaniam,et al. Wireless Sensor Network: A survey Computer Networks, 2003, 38(4): 393-422.

[2] H. Chan, A. Perrig, ACE: an emergent algorithm for highly uniform cluster formation, in: Proceedings of the First European Workshop Sensor Networks (EWSN), January 2004.

[3] P. Krishna, N.H. Vaidya, M. Chatterjee, D. Pradhan, A cluster-based approach for routing in dynamic networks, ACM SIGCOMM

Computer Communication Review 27 (2) (1997) 49-65.

[4] B. McDonald, T. Znati, Design and performance of a distributed dynamic clustering algorithm for Ad-

Hoc networks, in: Proceedings of the Annual Simulation Symposium, 2001.

[5] W.R. Heinzelman, A.P. Chandrakasan, H. Balakrishnan, An application-specific protocol architecture for wireless microsensor networks,IEEE Transactions on Wireless Communications 1 (4) (2002) 660670.

[6] W.R. Heinzelman, A.P. Chandrakasan, H. Balakrishnan, Energy efficient communication protocol for wireless microsensor networks, in Proceedings of the 33rd Hawaii International Conference on System Sciences (HICSS-33), January 2000.

[7] N. Kumar and V. S. Prabha, "Comparative analysis of energy-efficient cluster-based routing protocols for wireless sensor networks", Sensors and Transducers, vol. 142, Issue 7, (2012), pp. 23-32.

[8] S. Lindsey, C. S. Raghavendra, "PEGASIS: power efficient gathering in sensor information systems," in Proc. Of IEEE Aerospace Conference, pp. 1-6, 2002.

[9] Haitao Zhang, Shiwei Zhang* and Wenshao Bu, " A Clustering Routing Protocol for Energy Balance of Wireless Sensor Network based on Simulated Annealing and Genetic Algorithm", in International Journal of Hybrid Information Technology Vol.7, No.2 (2014), pp.71-82.

[10] A. Manjeshwar, D. P. Agrawal, "TEEN: a routing protocol for enhanced efficiency in wireless sensor networks," in Proc. of the 15th International Parallel and Distributed Processing Symposium, pp. 2009-2015, 2001.

[11] S. D. Muruganthan, D. C. F. Ma, B. Rollyi, A. Fapojuwo, "A centralized energy-efficient routing protocol for wireless sensor networks," IEEE Radio Communications, vol. 43, no. 3, pp. 8-13, 2005.

[12]Handy MJ, Hasse M, Timmermann D. Low energy adaptive clustering hierarchy with deterministic cluster-head selection [C]// Proc of the 4th IEEE Conf. on Mobile and Wireless Communications Networks. Stockholm, 2002:368-372.

[13] A. Manjeshwar and D. P. Agarwal. 2001. TEEN: a routing protocol for enhanced efficiency in wireless sensor networks. In 1st International Workshop on Parallel and Distributed Computing Issues in Wireless Networks and Mobile Computing.

[14] Smaragdakis G. Matta I. Bestavros A. A Stable Election Protocol for Clustered Heterogeneous Wireless Sensor Networks, Proceedings of the 2nd International Workshop on SANPA 2004,Massachusetts, U.S, 2004:1-11 
[15] E. Zeydan, D. Kivanc, C. Comaniciu, U. Tureli "Energy-efficient routing for correlated data in wireless sensor networks" Ad Hoc Networks, Volume 10, Issue 6, August 2012, Pages 962-975.

[16].V. Kumar, S. Jain and S. Tiwari "Impact of Node Density and Mobility on Scalable Routing Protocols in Mobile AdHoc Networks" Special Issue of International Journal of Computer Applications (0975 8887) on Communication Security, No.5 Mar.2012. 